\title{
PASSIVE FUZZY CONTROLLER DESIGN FOR PERTURBED NONLINEAR DRUM-BOILER SYSTEM WITH MULTIPLICATIVE NOISE
}

\author{
Cheung-Chieh Ku \\ Department of Electrical Engineering, National Taiwan Ocean University, \\ Pei-Hwa Huang \\ Department of Electrical Engineering, National Taiwan Ocean University, \\ Wen-Jer Chang \\ Department of Marine Engineering, National Taiwan Ocean University, Keelung, Taiwan, R.O.C, \\ wjchang@mail.ntou.edu.tw
}

Follow this and additional works at: https://jmstt.ntou.edu.tw/journal

Part of the Engineering Commons

\section{Recommended Citation}

Ku, Cheung-Chieh; Huang, Pei-Hwa; and Chang, Wen-Jer (2010) "PASSIVE FUZZY CONTROLLER DESIGN FOR PERTURBED NONLINEAR DRUM-BOILER SYSTEM WITH MULTIPLICATIVE NOISE," Journal of Marine Science and Technology: Vol. 18: Iss. 2, Article 7.

DOI: $10.51400 / 2709-6998.2320$

Available at: https://jmstt.ntou.edu.tw/journal/vol18/iss2/7

This Research Article is brought to you for free and open access by Journal of Marine Science and Technology. It has been accepted for inclusion in Journal of Marine Science and Technology by an authorized editor of Journal of Marine Science and Technology. 


\title{
PASSIVE FUZZY CONTROLLER DESIGN FOR PERTURBED NONLINEAR DRUM-BOILER SYSTEM WITH MULTIPLICATIVE NOISE
}

\author{
Cheung-Chieh $\mathrm{Ku}^{*}$, Pei-Hwa Huang*, and Wen-Jer Chang**
}

Key words: drum-boiler system, multiplicative noise, Itô's formula, passivity, T-S fuzzy model.

\begin{abstract}
This paper addresses the problem of designing robust passive fuzzy controller for uncertain nonlinear drum-boiler system with multiplicative noise. For this problem, the Takagi-Sugeno (T-S) fuzzy model is employed to represent the nonlinearities of boiler system to be an analyzed system. Based on the T-S fuzzy model, the sufficient stability conditions are developed as the Linear Matrix Inequality (LMI) problem by Itô's formula and Lyapunov function. Besides, the strictly input passive theory is employed to discuss the external disturbance effect on system and investigate attenuation performance for disturbance. With applying the proposed controller design technique, the perturbed nonlinear boiler system with multiplicative noise can be guaranteed to be mean square stable and strict input passive.
\end{abstract}

\section{INTRODUCTION}

In the many factories, the steam is widely used for hearting and keeping temperature. For this reason, the boiler becomes effective and essential steam generator to supply high quality steam. Hence, the stability and stabilization problems of nonlinear drum-boiler system $[2,7,13]$ are worth to be discussed and investigated. According to the stability and stabilization problems, the accurate model for describing the boiler systems must be proposed via valid method. Hence, the identification approach $[2,13]$ plays important role for describing the behaviors of boiler system to develop useful controller design techniques. Therefore, the Pellegrinetti and Bentsman [13] provided valid dynamic equation for representing the nonlinear drum-boiler system that derived by

Paper submitted 06/03/08; revised 12/23/08; accepted 05/13/09. Author for correspondence: Wen-Jer Chang (e-mail: wjchang@mail.ntou.edu.tw).

*Department of Electrical Engineering, National Taiwan Ocean University, Keelung, Taiwan, R.O.C.

**Department of Marine Engineering, National Taiwan Ocean University, Keelung, Taiwan, R.O.C. mathematical techniques with surveying many literatures. The proposed model of [7] was currently used in a real time simulator of a steam generation system for the purposes of evaluation of various control algorithms as well as for operator training. On the other hand, the model can be used to develop and investigate the variety techniques of control design $[9,14]$ by researchers and engineers. Unfortunately, the almost control design approaches [9, 14] were investigated by linear design method for representing local behaviors of nonlinear boiler system. In order to describe the nonlinearities of boiler system, Wang et al. [4] and Abdeldjebar et al. [1] applied the T-S fuzzy and Madani fuzzy approach respectively, to approximate the trajectories and develop the nonlinear controller design technique for boiler system.

Recently, the T-S fuzzy model approach [3, 5, 6, 19] provides a valid and useful tool to discuss the stability problems of nonlinear systems. With the development of T-S fuzzy model $[6,16]$, the robust control has been applied for nonlinear systems with internal perturbations. In generally, the perturbation effects on system were assumed as time-varying but bounded. Furthermore, the stochastic nonlinear systems have been described by T-S fuzzy model with multiplicative noise in $[10,17,19]$. The multiplicative noise term can be associated as the product of states and noises for describing the stochastic behaviors. From the multiplicative noise term, the stochastic systems could be referred to [8] as the "bilinear stochastic systems" that is a special case of the vast class of bilinear systems. With above illustrations, one can study stability issues of the uncertain nonlinear boiler system with multiplicative noise via the T-S fuzzy model approach. Besides, the fuzzy controller design can be carried out by Parallel Distributed Compensation (PDC) control technique [5]. In addition, the effect of external disturbance on boiler system is also considered in this paper for achieving attenuation performance.

For years, the passivity theorem $[5,11,12,18]$ proposes handy and useful tool for analyzing and synthesizing the stability of disturbed system with energy concept. The concept of passivity theorem in [12] can be associated as the energy change between the system and external disturbance. The strictly input passive concept of [12] is employed to discuss the effect of disturbance on system for discussing the attenuation per- 
formance in this paper. Besides, the relaxed concept of $[5,15]$ has been used to obtain new relaxed stability conditions for extending the stability region and relaxing the computational constraints. The above relaxed method in Tanaka and Wang [15] can be described as the only fired partial rules of fuzzy model instead of all rules. With applying the same relaxed concept as [15], the stability of disturbed nonlinear systems in [5] can be guaranteed in Lyapunov stable and strictly input passive. Accordingly, the stability and stabilization problems of uncertain T-S fuzzy model with multiplicative noise are discussed with relaxed concept in this paper.

The main contribution of this paper is to develop relaxed stability conditions for analyzing and synthesizing the stability of nonlinear drum-boiler system with uncertainties and multiplicative noise via the T-S fuzzy model approach. For building the T-S fuzzy model, the Teizeira-Zak's formula in [16] is employed to obtain the linear sub-systems and membership function with determined operation points. Furthermore, the robust control and stochastic behaviors are taken account of modeling error, perturbation, operating varying and environment change. Besides, the external disturbance is discussed by passivity theorem for attenuation performance. Based on the derived stability conditions, the PDC-based fuzzy controller can be obtained via combining feedback gains and membership function. By using the proposed design method, the perturbed nonlinear drum-boiler system can achieve the desired targets. Finally, the simulation results of boiler system with designed fuzzy controller are proposed to manifest the proposed design technique.

\section{SYSTEM STATEMENTS AND PROBLEM FORMULATIONS}

In this section, the T-S fuzzy model of boiler system was proposed with multiplicative noise and time-varying parameters to be the considered system. In the Fig. 1, the main process of nonlinear drum-boiler system can be shown with inputs and outputs.

From Fig. 1, the interesting measured outputs can be stated as measured drum pressure, excess oxygen level, drum water level and steam flow rate, to provide the available data for engineers. In order to maintain the smooth outputs and safety of system, the control input can be considered as the fuel flow, air flow and feed water rates while sensing steam pressure, actual fuel rate, drum water level, actual feed water flow rate, steam flow rate, steam temperature, actual air flow rate and oxygen level in the flue gas. Then, the dynamic equation of boiler system was presented as follows:

$$
\dot{w}_{1}(t)=-0.00478 w_{4}(t) w_{1}^{9 / 8}(t)+0.28 p_{1}(t)-0.01348 p_{3}(t)
$$

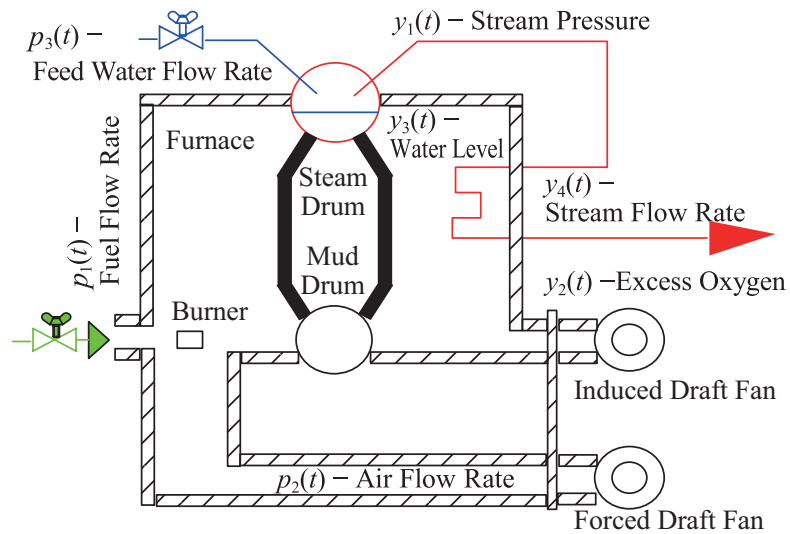

Fig. 1. Industrial Steam Boiler.

$$
\begin{gathered}
\dot{w}_{2}(t)=0.1540357 w_{2}(t)+0.1 v(t)+\left(103.5462 p_{2}(t)\right. \\
\left.-107.4835 p_{1}(t)-1.9515 p_{1}(t) w_{2}(t)\right) / \\
\\
\left(29.04 p_{2}(t)-1.824 p_{1}(t)\right) \\
\dot{w}_{3}(t)=-0.00533176 w_{1}(t)-0.025195 w_{4}(t) w_{1}(t) \\
+0.7317058 p_{3}(t) \\
\dot{w}_{4}(t)=-0.04 w_{4}(t)+0.029988 p_{1}(t)+0.018088 \\
y_{1}(t)=14.214 w_{1}(t) \\
y_{2}(t)=w_{2}(t)
\end{gathered}
$$

$$
\begin{aligned}
y_{3}(t)= & -0.1048569 w_{1}(t)+0.15479 w_{3}(t)+0.495 w_{4}(t) w_{1}(t) \\
& -0.2 p_{3}(t)+1.272 p_{1}(t)-\left(324212.78 w_{1}(t)+99556.25\right) \\
& \times\left(1-0.0012 w_{3}(t)\right) /\left(w_{3}(t)\left(w_{1}(t)-1704.5\right)\right)-103.74
\end{aligned}
$$

$$
y_{4}(t)=\left(0.85663 w_{4}(t)-0.18128\right) w_{1}(t)
$$

where $w_{1}(t)$ is the drum pressure state $\left(\mathrm{kgf} / \mathrm{cm}^{2}\right) ; w_{2}(t)$ is the excess oxygen level (percent); $w_{3}(t)$ is the system fluid density $\left(\mathrm{kg} / \mathrm{m}^{3}\right) ; w_{4}(t)$ is the exogenous variable related to the load disturbance intensity $(0-1) ; y_{1}(t), y_{2}(t), y_{3}(t)$ and $y_{4}(t)$ are the measured outputs for drum pressure (PSI), excess oxygen level (percent), drum water level (in) and steam flow rate $(\mathrm{kg} / \mathrm{s})$, respectively; $p_{1}(t), p_{2}(t)$ and $p_{3}(t)$ are the fuel, air and feed water level rate inputs which take values between $0-1 ; v(t)$ is the external disturbance input. Form [4], the equilibrium point of (1) can be obtained such as: 


$$
\begin{gathered}
{\left[\begin{array}{cccc}
w_{1}(t) & w_{2}(t) & w_{3}(t) & w_{4}(t) \\
p_{1}(t) & p_{2}(t) & p_{3}(t)
\end{array}\right]_{e p}} \\
\quad=\left[\begin{array}{cccc}
22.5 & 2.5 & 621.17 & 0.8374 \\
0.5138 & 0.5064 & 0.8127
\end{array}\right]
\end{gathered}
$$

Based on the equilibrium points in (3), the new states and inputs can be obtained as follows:

$$
\begin{aligned}
& {\left[\begin{array}{cccc}
w_{1}(t) & w_{2}(t) & w_{3}(t) & w_{4}(t) \\
p_{1}(t) & p_{2}(t) & p_{3}(t)
\end{array}\right]} \\
& =\left[\begin{array}{cccc}
x_{1}(t)+22.5 & x_{2}(t)+2.5 & x_{3}(t)+621.17 & x_{4}(t)+0.8374 \\
u_{1}(t)+0.5138 & u_{2}(t)+0.5064 & u_{3}(t)+0.8127
\end{array}\right]
\end{aligned}
$$

Substituting (4) into (1), we have following new dynamic equation to represent the original boiler system (1).

$$
\begin{aligned}
\dot{x}_{1}(t)= & -0.00478\left(x_{4}(t)+0.8374\right)\left(x_{1}(t)+22.5\right)^{9 / 8}+0.28\left(u_{1}(t)\right. \\
& +0.5138)-0.01348\left(u_{3}(t)+0.8127\right)
\end{aligned}
$$

$$
\begin{aligned}
\dot{x}_{2}(t)= & 0.1540357\left(x_{2}(t)+2.5\right)+0.1 v(t)+\left(1 0 3 . 5 4 6 2 \left(u_{2}(t)\right.\right. \\
& +0.5064)-107.4835\left(u_{1}(t)+0.5138\right)-1.9515\left(u_{1}(t)\right. \\
& \left.+0.5138)\left(x_{2}(t)+2.5\right)\right) /\left(29.04\left(x_{2}(t)+2.5\right)-1.824\right. \\
& \left.\times\left(u_{1}(t)+0.5138\right)\right)
\end{aligned}
$$

$$
\begin{aligned}
\dot{x}_{3}(t)= & -0.00533176\left(x_{1}(t)+22.5\right)-0.025195\left(x_{4}(t)+0.8374\right) \\
& \times\left(x_{1}(t)+22.5\right)+0.7317058\left(u_{3}(t)+0.8127\right)
\end{aligned}
$$$$
\dot{x}_{4}(t)=-0.04\left(x_{4}(t)+0.8374\right)+0.029988\left(u_{1}(t)+0.5138\right)
$$$$
+0.018088
$$

Furthermore, by setting $u_{1}(t)=u_{2}(t)=u_{3}(t)=v(t)=0$, the Teixeira-Zak's linearization technique in [16] was applied to obtain the linear sub-systems from (5) with following three operation points.

$$
\begin{aligned}
x^{o p-1} & =\left[\begin{array}{llll}
-22.5 & 0 & 0 & 0
\end{array}\right]^{\mathrm{T}}, \quad x^{o p-2}=\left[\begin{array}{llll}
0 & 0 & 0 & 0
\end{array}\right]^{\mathrm{T}} \text { and } \\
x^{o p-3} & =\left[\begin{array}{llll}
22.5 & 0 & 0 & 0
\end{array}\right]^{\mathrm{T}}
\end{aligned}
$$

In which, the $x^{o p-2}$ is an equilibrium point of (5). And then, the T-S fuzzy model can be obtained with three fuzzy rules, such as:

Plant Rule ${ }^{1}$ IF $x_{1}(t)$ is -22.5 THEN

$$
\dot{x}(t)=\mathbf{A}_{1} x(t)+\mathbf{B}_{1} u(t)+\mathbf{E}_{1} v(t)
$$

Plant Rule ${ }^{1}$ IF $x_{1}(t)$ is 0 THEN

$$
\dot{x}(t)=\mathbf{A}_{2} x(t)+\mathbf{B}_{2} u(t)+\mathbf{E}_{2} v(t)
$$

Plant Rule ${ }^{1}$ IF $x_{1}(t)$ is 22.5 THEN

$$
\dot{x}(t)=\mathbf{A}_{3} x(t)+\mathbf{B}_{3} u(t)+\mathbf{E}_{3} v(t)
$$

where $x(t)=\left[\begin{array}{llll}x_{1}^{\mathrm{T}}(t) & x_{2}^{\mathrm{T}}(t) & x_{3}^{\mathrm{T}}(t) & x_{4}^{\mathrm{T}}(t)\end{array}\right]^{\mathrm{T}}$,

$$
u(t)=\left[\begin{array}{lll}
u_{1}^{\mathrm{T}}(t) & u_{2}^{\mathrm{T}}(t) & u_{3}^{\mathrm{T}}(t)
\end{array}\right]
$$

$\mathbf{B}_{1}=\mathbf{B}_{2}=\mathbf{B}_{3}=\left[\begin{array}{ccc}0.28 & 0 & -0.0135 \\ -8.2117 & 8.3317 & 0 \\ 0 & 0 & 0.7317 \\ 0.03 & 0 & 0\end{array}\right]$,

$\mathbf{E}_{1}=\mathbf{E}_{2}=\mathbf{E}_{3}=\left[\begin{array}{c}0 \\ 0.1 \\ 0 \\ 0\end{array}\right], \quad \mathbf{A}_{1}=\left[\begin{array}{cccc}-0.0059 & 0 & 0 & 0 \\ 0 & 0.0812 & 0 & 0 \\ -0.0264 & 0 & 0 & 0 \\ 0 & 0 & 0 & -0.04\end{array}\right]$,

$\mathbf{A}_{2}=\left[\begin{array}{cccc}-0.0066 & 0 & 0 & -0.1587 \\ 0 & 0.0812 & 0 & 0 \\ -0.0264 & 0 & 0 & -0.5669 \\ 0 & 0 & 0 & -0.04\end{array}\right]$ and

$\mathbf{A}_{3}=\left[\begin{array}{cccc}-0.007 & 0 & 0 & -0.3462 \\ 0 & 0.0812 & 0 & 0 \\ -0.0264 & 0 & 0 & -1.1338 \\ 0 & 0 & 0 & -0.04\end{array}\right]$. Besides, with the op-

eration points, the three triangular functions were provided to structure the membership function that was presented in Fig. 2.

In addition, we added the multiplicative noise and uncertainty terms to represent the operating changing and modeling errors for simulating the real operating conditions. And, the controlled output was added for achieving the disturbance attenuation performance, too. With the above considering, the final output of overall T-S fuzzy model can be inferred as follows: 


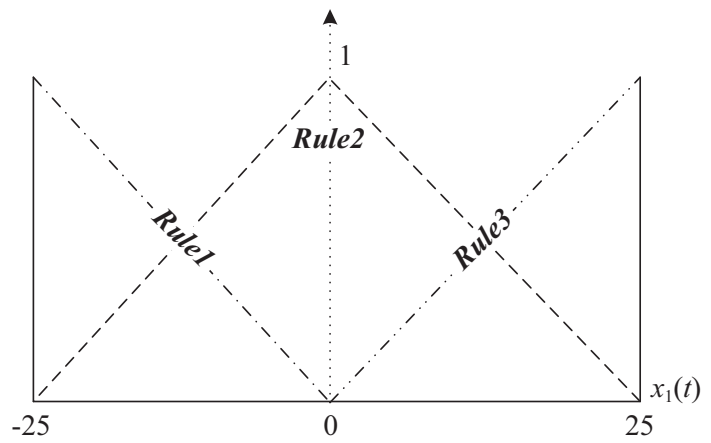

Fig. 2. Membership function of $x_{1}(t)$.

$\dot{x}(t)=\sum_{i=1}^{3} h_{i}\left(x_{1}(t)\right)\left\{\left(f_{i}(t)+\Delta f_{i}(t)\right)+\left(g_{i}(t)+\Delta g_{i}(t)\right) \beta_{i}(t)\right\}$

$z(t)=\sum_{i=1}^{3} h_{i}\left(x_{1}(t)\right)\left\{\mathbf{C}_{i} x(t)+\mathbf{D}_{i} v(t)\right\}$

where

$$
\begin{aligned}
& f_{i}(t)=\mathbf{A}_{i} x(t)+\mathbf{B}_{i} u(t)+\mathbf{E}_{i} v(t) \text { and } \\
& \Delta f_{i}(t)=\mathbf{H} \Delta(t)\left(\mathbf{R}_{i} x(t)+\mathbf{R}_{\mathbf{B} i} u(t)+\mathbf{R}_{\mathbf{E} i} v(t)\right), \\
& g(t)=\overline{\mathbf{A}}_{i} x(t)+\overline{\mathbf{B}}_{i} u(t)+\overline{\mathbf{E}}_{i} v(t) \text { and } \\
& \Delta g(t)=\overline{\mathbf{H}} \bar{\Delta}(t)\left(\overline{\mathbf{R}}_{i} x(t)+\overline{\mathbf{R}}_{\mathbf{B} i} u(t)+\overline{\mathbf{R}}_{\mathbf{E} i} v(t)\right),
\end{aligned}
$$

for $i=1,2,3 . \quad \sum_{i=1}^{3} h_{i}\left(x_{1}(t)\right)=1$ and $h_{i}\left(x_{1}(t)\right) \geq 0$ which is the grade of membership function, $z(t)$ is the controlled output, $\beta_{i}(t)$ are a scalar zero-mean Gaussian white noise process with unit covariance, $\Delta(t)$ and $\bar{\Delta}(t)$ are the time-varying function with $\Delta(t) \Delta(t) \leq \mathbf{I}$ and $\bar{\Delta}(t) \bar{\Delta}(t) \leq \mathbf{I}$. Besides, in the following, we let $h_{\mathrm{i}}=h_{\mathrm{i}}\left(x_{1}(t)\right)$ and $h_{\mathrm{j}}=h_{\mathrm{j}}\left(x_{1}(t)\right)$ to simplify unnecessary descriptions of this paper. With using the PDC concept, the fuzzy controller can be carried out via the linear feedback gain with the same fuzzy sets of plant. Hence, the PDC-based fuzzy controller was proposed as follows:

$$
u(t)=\sum_{i=1}^{3} h_{i}\left(\mathbf{F}_{i} x(t)\right)
$$

Substituting the (10) into (8a), one can find the closed-loop uncertain T-S fuzzy model with multiplicative noise, such as:

$$
\begin{aligned}
& \dot{x}(t)=\sum_{i=1, j=1}^{3} h_{i} h_{j}\left\{f_{i j}(t)+g_{i j}(t) \beta(t)\right\} \\
& =\sum_{i=1}^{3} h_{i}^{2}\left\{f_{i i}(t)+g_{i i}(t) \beta(t)\right\}+2 \sum_{i=1, j<i}^{3} h_{i} h_{j}\left\{f_{i j}(t)+g_{i j}(t) \beta(t)\right\}
\end{aligned}
$$

where $f_{i i}(t)=\mathbf{G}_{i i} x(t)+\mathbf{E}_{i} v(t)+\mathbf{H} \Delta(t)\left(\mathbf{R}_{i i} x(t)+\mathbf{R}_{\mathbf{E} i} v(t)\right)$,

$g_{i i}(t)=\overline{\mathbf{G}}_{i i} x(t)+\overline{\mathbf{E}}_{i} v(t)+\overline{\mathbf{H}} \bar{\Delta}(t)\left(\overline{\mathbf{R}}_{i i} x(t)+\overline{\mathbf{R}}_{\mathbf{E} i} v(t)\right)$

$$
\begin{aligned}
f_{i j}(t)= & \left(\mathbf{G}_{i j}+\mathbf{G}_{j i}\right) x(t)+\mathbf{E}_{i j} v(t)+\mathbf{H} \Delta(t)\left(\left(\mathbf{R}_{i j}+\mathbf{R}_{j i}\right) x(t)\right. \\
& \left.+\mathbf{R}_{\mathbf{E} i j} v(t)\right)
\end{aligned}
$$

$g_{i j}(t)=\left(\overline{\mathbf{G}}_{i j}+\overline{\mathbf{G}}_{j i}\right) x(t)+\overline{\mathbf{E}}_{i j} v(t)+\overline{\mathbf{H}} \bar{\Delta}(t)\left(\left(\overline{\mathbf{R}}_{i j}+\overline{\mathbf{R}}_{j i}\right) x(t)\right.$

$$
\left.+\overline{\mathbf{R}}_{\mathbf{E} i j} v(t)\right)
$$

and $\mathbf{G}_{i i}=\mathbf{A}_{i}+\mathbf{B}_{i} \mathbf{F}_{i}, \quad \mathbf{R}_{i i}=\mathbf{R}_{i}+\mathbf{R}_{\mathbf{B} i} \mathbf{F}_{i}, \overline{\mathbf{G}}_{i i}=\overline{\mathbf{A}}_{i}+\overline{\mathbf{B}}_{i} \mathbf{F}_{i}$,

$$
\begin{aligned}
& \overline{\mathbf{R}}_{i i}=\overline{\mathbf{R}}_{i}+\overline{\mathbf{R}}_{\mathbf{B} i} \mathbf{F}_{i}, \quad \mathbf{G}_{i j}=\frac{\mathbf{A}_{i}+\mathbf{B}_{i} \mathbf{F}_{j}}{2}, \quad \mathbf{E}_{i j}=\frac{\mathbf{E}_{i}+\mathbf{E}_{j}}{2}, \\
& \mathbf{R}_{\mathbf{E} i j}=\frac{\mathbf{R}_{\mathbf{E} i}+\mathbf{R}_{\mathbf{E} j}}{2}, \quad \mathbf{R}_{i j}=\frac{\mathbf{R}_{i}+\mathbf{R}_{\mathbf{B} i} \mathbf{F}_{j}}{2}, \overline{\mathbf{G}}_{i j}=\frac{\overline{\mathbf{A}}_{i}+\overline{\mathbf{B}}_{i} \mathbf{F}_{j}}{2}, \\
& \overline{\mathbf{E}}_{i j}=\frac{\overline{\mathbf{E}}_{i}+\overline{\mathbf{E}}_{j}}{2}, \quad \overline{\mathbf{R}}_{\mathbf{E} i j}=\frac{\overline{\mathbf{R}}_{\mathbf{E} i}+\overline{\mathbf{R}}_{\mathbf{E} j}}{2}, \quad \overline{\mathbf{R}}_{i j}=\frac{\overline{\mathbf{R}}_{i}+\overline{\mathbf{R}}_{\mathbf{B} i} \overline{\mathbf{F}}_{j}}{2}
\end{aligned}
$$

With the energy concept, the passivity theory provides a useful and effective tool to design the controller to achieve the energy constraints for the closed-loop systems. In order to constrain the disturbance energy, we choose the strict input passive of the passive theory in this paper. And, the strict input passivity can be introduced as following definition.

\section{Definition 1 [5]}

If there exists $\gamma>0$ for satisfying following inequality, then the system is called the strictly input passive with the disturbance $v(t)$ and output $z(t)$ such as:

$$
E\left\{2 \int_{0}^{t} z^{\mathrm{T}}(t) v(t) d t\right\} \geq E\left\{\gamma \int_{0}^{t} v^{\mathrm{T}}(t) v(t) d t\right\}
$$

for all terminal time of control $t>0$. In which, $E\{\bullet\}$ denotes the expected value of $\bullet$. 
Furthermore, the following mathematical techniques were introduced for developing the sufficient stability conditions in this paper.

\section{Lemma $1[10,19]$}

Given real compatible dimensions matrices $\mathbf{A}, \mathbf{H}$ and $\mathbf{R}$ for any matrix $\mathbf{X}>0, \varepsilon>0, \Delta(t)$ with $\Delta^{\mathrm{T}}(t) \Delta(t) \leq \mathbf{I}$, one can find the two results as follows:

a). $\mathbf{H} \Delta(t) \mathbf{R}+\mathbf{R}^{\mathrm{T}} \Delta^{\mathrm{T}}(t) \mathbf{H}^{\mathrm{T}} \leq \varepsilon \boldsymbol{\varepsilon} \mathbf{H} \mathbf{H}^{\mathrm{T}}+\boldsymbol{\varepsilon}^{-1} \mathbf{R}^{\mathrm{T}} \mathbf{R}$

b). If $\mathbf{X}-\varepsilon \Delta(t) \Delta^{T}(t) \geq 0$ hold, then

$$
\begin{aligned}
(\mathbf{A} & +\mathbf{H} \Delta(t) \mathbf{R})^{\mathrm{T}} \mathbf{X}^{-1}(\mathbf{A}+\mathbf{H} \Delta(t) \mathbf{R}) \\
& \leq \mathbf{A}^{\mathrm{T}}\left(\mathbf{X}-\varepsilon \mathbf{H H}^{T}\right)^{-1} \mathbf{A}+\varepsilon^{-1} \mathbf{R}^{\mathrm{T}} \mathbf{R}
\end{aligned}
$$

\section{Lemma $2[5,15]$}

Based on the rules fired criterion, the following inequality can be obtained when the number of fired rule is less or equal to $s$, where $1<s \leq r$ and $r$ is number of fuzzy rule, for all $t$.

$$
\sum_{\mathrm{i}=1}^{\mathrm{r}} h_{\mathrm{i}}^{2}-\frac{1}{s-1} \sum_{\mathrm{i}=1, \mathrm{i}<\mathrm{j}}^{\mathrm{r}} 2 h_{\mathrm{i}} h_{\mathrm{j}} \geq 0
$$

where $h_{\mathrm{i}} \geq 0$ and $\sum_{\mathrm{i}=1}^{\mathrm{r}} h_{\mathrm{i}}=1$ for all $t$.

The results of Lemma 2 follows directly from Tanaka and Wang [15]. By using the above definition and lemmas, the relaxed stability conditions of closed-loop uncertain T-S fuzzy model with multiplicative noise (11) were developed by the Itô's formula and Lyapunov function in the following theorem.

\section{DEVELOPMENT OF RELAXED STABILITY CONDITIONS}

In this section, the stability and stabilization problems of uncertain T-S fuzzy model with multiplicative term were discussed for guaranteeing the stability of nonlinear drum-boiler system. According to the relaxed concept of Lemma 2, the region of feasible solutions can be extended to satisfy the stability condition and design the fuzzy controller easily.

\section{Theorem 1}

Given dissipative rate $\gamma>0$ and number of fired rule $s>1$, if there exits $\mathbf{P}=\mathbf{P}^{\mathrm{T}}>0, \mathbf{Q}=\mathbf{Q}^{\mathrm{T}}>0, \varepsilon_{i i}>0, \varepsilon_{i j}>0$ and feedback gain $\mathbf{F}_{i}$ to satisfy the following relaxed stability conditions, then the uncertain T-S fuzzy model with multiplicative noise is mean square stable and strictly input passive.

$$
\boldsymbol{\Lambda} \equiv\left[\begin{array}{cc}
\boldsymbol{\Lambda}_{11}+(s-1) \mathbf{Q} & \boldsymbol{\Lambda}_{12}-\mathbf{C}_{i}^{\mathrm{T}} \\
* & \boldsymbol{\Lambda}_{22}+\gamma \mathbf{I}-\mathbf{D}_{i}^{\mathrm{T}}-\mathbf{D}_{i}
\end{array}\right] \leq 0, \quad i=j
$$

$$
\begin{aligned}
& \tilde{\boldsymbol{\Lambda}} \equiv\left[\begin{array}{cc}
\tilde{\boldsymbol{\Lambda}}_{11}-\mathbf{Q} & \tilde{\boldsymbol{\Lambda}}_{12}-\left(\mathbf{C}_{i}+\mathbf{C}_{j}\right)^{\mathrm{T}} / 2 \\
* & \tilde{\boldsymbol{\Lambda}}_{22}+\gamma \mathbf{I}-\left(\mathbf{D}_{i}^{\mathrm{T}}+\mathbf{D}_{i}+\mathbf{D}_{j}^{\mathrm{T}}+\mathbf{D}_{j}\right)^{\mathrm{T}} / 2
\end{array}\right] \leq 0, \\
& i \neq j
\end{aligned}
$$

where * means the transposed elements of matrices for symmetric position, I denotes compatible dimension identity matrix and

$$
\begin{aligned}
& \boldsymbol{\Lambda}_{11}=\mathbf{G}_{i i}^{\mathrm{T}} \mathbf{P}+\mathbf{P G}_{i i}+2 \mathbf{P} \mathbf{H} \mathbf{H}^{\mathrm{T}} \mathbf{P}+\varepsilon_{i i}^{-1} \mathbf{R}_{i i}^{\mathrm{T}} \mathbf{R}_{i i}+\overline{\mathbf{G}}_{i i}^{\mathrm{T}} \mathbf{T}_{i i} \overline{\mathbf{G}}_{i i}, \\
& +\mathcal{E}_{i i}^{-1} \overline{\mathbf{R}}_{i i}^{\mathrm{T}} \overline{\mathbf{R}}_{i i}, \\
& \boldsymbol{\Lambda}_{12}=\mathbf{P} \mathbf{E}_{i}+\overline{\mathbf{G}}_{i i}^{\mathrm{T}} \mathbf{T}_{i i} \overline{\mathbf{E}}_{i}+\mathcal{\varepsilon}_{i i}^{-1} \overline{\mathbf{R}}_{i i}^{\mathrm{T}} \overline{\mathbf{R}}_{\mathbf{E} i}, \\
& \boldsymbol{\Lambda}_{22}=\varepsilon_{i i}^{-1} \mathbf{R}_{\mathbf{E} i}^{\mathrm{T}} \mathbf{R}_{\mathbf{E} i}+\overline{\mathbf{E}}_{i}^{\mathrm{T}} \mathbf{T}_{i i} \overline{\mathbf{E}}_{i}+\varepsilon_{i i}^{-1} \overline{\mathbf{R}}_{\mathbf{E} i}^{\mathrm{T}} \overline{\mathbf{R}}_{\mathbf{E} i} \\
& \tilde{\boldsymbol{\Lambda}}_{11}=\left(\mathbf{G}_{i j}+\mathbf{G}_{j i}\right)^{\mathrm{T}} \mathbf{P}+\mathbf{P}\left(\mathbf{G}_{i j}+\mathbf{G}_{j i}\right)+4 \mathbf{P} \mathbf{H} \mathbf{H}^{\mathrm{T}} \mathbf{P}+\varepsilon_{i j}^{-1} \mathbf{Y}_{i j}^{\mathrm{T}} \mathbf{Y}_{i j} \\
& +\overline{\mathbf{M}}_{i j}^{\mathrm{T}} \mathbf{T}_{i j} \overline{\mathbf{M}}_{i j}+\varepsilon_{i j}^{-1} \overline{\mathbf{Y}}_{i j}^{\mathrm{T}} \overline{\mathbf{Y}}_{i j} \text {, } \\
& \tilde{\boldsymbol{\Lambda}}_{21}=\mathbf{P} \mathbf{E}_{i j}+\overline{\mathbf{M}}_{i j}^{\mathrm{T}} \mathbf{T}_{i j} \overline{\mathbf{M}}_{\mathbf{E} i j}+\varepsilon_{i j}^{-1} \overline{\mathbf{Y}}_{i j}^{\mathrm{T}} \overline{\mathbf{Y}}_{\mathbf{E} i j}, \\
& \tilde{\mathbf{\Lambda}}_{22}=\varepsilon_{i j}^{-1} \mathbf{Y}_{\mathbf{E} i j}^{\mathrm{T}} \mathbf{Y}_{\mathbf{E} i j}+2 \overline{\mathbf{M}}_{\mathbf{E} i j}^{\mathrm{T}} \mathbf{T}_{i j} \overline{\mathbf{M}}_{\mathbf{E} i j}+\varepsilon_{i j}^{-1} \overline{\mathbf{Y}}_{\mathbf{E} i j}^{\mathrm{T}} \overline{\mathbf{Y}}_{\mathbf{E} i j},
\end{aligned}
$$

and $\overline{\mathbf{M}}_{i j}^{\mathrm{T}}=\left[\begin{array}{ll}\overline{\mathbf{G}}_{i j}^{\mathrm{T}} & \overline{\mathbf{G}}_{j i}^{\mathrm{T}}\end{array}\right], \overline{\mathbf{Y}}_{i j}^{\mathrm{T}}=\left[\begin{array}{ll}\overline{\mathbf{R}}_{i j}^{\mathrm{T}} & \overline{\mathbf{R}}_{j i}^{\mathrm{T}}\end{array}\right], \quad \mathbf{Y}_{i j}^{\mathrm{T}}=\left[\begin{array}{ll}\mathbf{R}_{i j}^{\mathrm{T}} & \mathbf{R}_{j i}^{\mathrm{T}}\end{array}\right]$, $\overline{\mathbf{M}}_{\mathbf{E} i j}^{\mathrm{T}}=\left[\begin{array}{ll}\overline{\mathbf{E}}_{i}^{\mathrm{T}} / 2 & \overline{\mathbf{E}}_{j}^{\mathrm{T}} / 2\end{array}\right], \quad \mathbf{Y}_{\mathbf{E} i j}^{\mathrm{T}}=\left[\begin{array}{ll}\mathbf{R}_{\mathbf{E} i}^{\mathrm{T}} / 2 & \mathbf{R}_{\mathbf{E} j}^{\mathrm{T}} / 2\end{array}\right]$, $\overline{\mathbf{Y}}_{\mathbf{E} i j}^{\mathrm{T}}=\left[\begin{array}{ll}\overline{\mathbf{R}}_{\mathbf{E} i}^{\mathrm{T}} / 2 & \overline{\mathbf{R}}_{\mathbf{E} j}^{\mathrm{T}} / 2\end{array}\right], \mathbf{T}_{i i}=\left(\mathbf{P}_{1}^{-1}-\varepsilon_{i i} \overline{\mathbf{H}} \overline{\mathbf{H}}^{\mathrm{T}}\right)^{-1}$ and

$$
\mathbf{T}_{i j}=\operatorname{diag}\left\{\left(\mathbf{P}^{-1}-\varepsilon_{i j} \overline{\mathbf{H}} \overline{\mathbf{H}}^{\mathrm{T}}\right)^{-1},\left(\mathbf{P}^{-1}-\varepsilon_{i j} \overline{\mathbf{H}} \overline{\mathbf{H}}^{\mathrm{T}}\right)^{-1}\right\} .
$$

in which $\operatorname{diag}\{\bullet\}$ denotes diagonal matrix with element $\bullet$.

\section{Proof:}

Let choose the Lyapunov function $V(x(t))=x^{\mathrm{T}}(t) \mathbf{P} x(t)$. And then, the differentiation of $V(x(t))$ along the trajectories of (11) can be obtained by the Itô's formula in Eli et al. [17], such as:

$$
\frac{d}{d t} V(x(t))=L V(x(t))+2 \sum_{i=1, j=1}^{3} h_{i} h_{j}\left\{x^{\mathrm{T}}(t) \mathbf{P}_{1} g_{i j}(t) \dot{\beta}_{i}\right\}
$$

in which, 


$$
\begin{gathered}
L V(x(t))=\sum_{i=1}^{3} h_{i}^{2}\left\{f_{i i}^{\mathrm{T}}(t) \mathbf{P} x(t)+x^{\mathrm{T}}(t) \mathbf{P} f_{i i}(t)\right. \\
\left.+\left(g_{i i}^{\mathrm{T}}(t) \mathbf{P} g_{i i}^{\mathrm{T}}(t)\right)\right\}+\sum_{i=1, j<i}^{3} h_{i} h_{j}\left\{f_{i j}^{\mathrm{T}}(t) \mathbf{P} x(t)\right. \\
\left.+x^{\mathrm{T}}(t) \mathbf{P} f_{i j}^{\mathrm{T}}(t)+\left(g_{i j}^{\mathrm{T}}(t) \mathbf{P} g_{i j}^{\mathrm{T}}(t)\right)\right\}
\end{gathered}
$$

Applying the Lemma 1, the uncertain matrices of (22) can be converted as follows:

$$
\begin{aligned}
& 2 \boldsymbol{\varphi}_{i i}^{\mathrm{T}} \Delta_{\Delta}^{\mathrm{T}}(t) \mathbf{H}_{\mathbf{H}}^{\mathrm{T}} \mathbf{P} x(t) \leq 2 \varepsilon_{i i} x^{\mathrm{T}}(t) \mathbf{P} \mathbf{H} \mathbf{H}^{\mathrm{T}} \mathbf{P} x(t)+\varepsilon_{i i}^{-1} \boldsymbol{\varphi}_{i i}^{\mathrm{T}} \boldsymbol{\varphi}_{i i} \\
& 2 \boldsymbol{\varphi}_{i j}^{\mathrm{T}} \Delta_{\Delta}^{\mathrm{T}}(t) \mathbf{H}_{\mathbf{H H}}^{\mathrm{T}} \mathbf{P} x(t) \leq 4 \varepsilon_{i j} x^{\mathrm{T}}(t) \mathbf{P} \mathbf{H} \mathbf{H}^{\mathrm{T}} \mathbf{P} x(t)+\varepsilon_{i j}^{-1} \boldsymbol{\varphi}_{i j}^{\mathrm{T}} \boldsymbol{\varphi}_{i j} \\
& \bar{x}^{\mathrm{T}}\left(\boldsymbol{\Psi}_{i i}+\overline{\mathbf{H}} \bar{\Delta}(t) \overline{\boldsymbol{\varphi}}_{i i}\right)^{\mathrm{T}} \mathbf{P}\left(\boldsymbol{\Psi}_{i i}+\overline{\mathbf{H}} \bar{\Delta}(t) \overline{\boldsymbol{\varphi}}_{i i}\right) \bar{x} \\
& \quad \leq \bar{x}^{\mathrm{T}}\left(\boldsymbol{\Psi}_{i i}^{\mathrm{T}} \mathbf{T}_{i i} \boldsymbol{\Psi}_{i i}+\varepsilon_{i i}^{-1} \overline{\boldsymbol{\varphi}}_{i i}^{\mathrm{T}} \overline{\boldsymbol{\varphi}}_{i i}\right) \bar{x} \\
& \bar{x}^{\mathrm{T}}\left(\boldsymbol{\Psi}_{i j}+\overline{\mathbf{H}}_{\mathbf{H}} \bar{\Delta}_{\Delta}(t) \overline{\boldsymbol{\varphi}}_{i j}\right)^{\mathrm{T}} \mathbf{P}_{\mathbf{P}}\left(\boldsymbol{\Psi}_{i j}+\overline{\mathbf{H}}_{\mathbf{H}} \bar{\Delta}_{\Delta}(t) \overline{\boldsymbol{\varphi}}_{i j}\right) \bar{x} \\
& \quad \leq \bar{x}^{\mathrm{T}}\left(\boldsymbol{\Psi}_{i j}^{\mathrm{T}} \mathbf{T}_{i j} \boldsymbol{\Psi}_{i j}+\varepsilon_{i j}^{-1} \overline{\boldsymbol{\varphi}}_{i j}^{\mathrm{T}} \overline{\boldsymbol{\varphi}}_{i j}\right) \bar{x}
\end{aligned}
$$

where $\Delta_{\Delta}(t)=\operatorname{diag}\{\Delta(t), \Delta(t)\}, \bar{\Delta}_{\Delta}(t)=\operatorname{diag}\{\bar{\Delta}(t), \bar{\Delta}(t)\}$, $\bar{x}^{\mathrm{T}}=\left[\begin{array}{ll}x^{\mathrm{T}}(t) & v^{\mathrm{T}}(t)\end{array}\right], \quad \boldsymbol{\Psi}_{i i}=\left[\begin{array}{ll}\overline{\mathbf{G}}_{i i} & \overline{\mathbf{E}}_{i}\end{array}\right]$,

$\boldsymbol{\psi}_{i j}=\left[\begin{array}{ll}\overline{\mathbf{M}}_{i j} & \overline{\mathbf{M}}_{\mathbf{E} i j}\end{array}\right], \boldsymbol{\varphi}_{i i}^{\mathrm{T}}=\left[\begin{array}{ll}x^{\mathrm{T}}(t) \mathbf{R}_{i i}^{\mathrm{T}} & v^{\mathrm{T}}(t) \mathbf{R}_{\mathbf{E} i}^{\mathrm{T}}\end{array}\right]$,

$\boldsymbol{\varphi}_{i j}^{\mathrm{T}}=\left[\begin{array}{ll}x^{\mathrm{T}}(t) \mathbf{Y}_{i j}^{\mathrm{T}} & v^{\mathrm{T}}(t) \mathbf{Y}_{\mathbf{E} i j}^{\mathrm{T}}\end{array}\right], \overline{\boldsymbol{\varphi}}_{i i}=\left[\begin{array}{ll}\overline{\mathbf{R}}_{i i} & \overline{\mathbf{R}}_{\mathbf{E} i}\end{array}\right]$,

$\overline{\boldsymbol{\varphi}}_{i j}=\left[\begin{array}{ll}\overline{\mathbf{Y}}_{i j} & \overline{\mathbf{Y}}_{\mathbf{E} i j}\end{array}\right], \quad \mathbf{H}_{\mathbf{H}}=\left[\begin{array}{ll}\mathbf{H} & \mathbf{H}\end{array}\right], \overline{\mathbf{H}}_{\mathbf{H}}=\left[\begin{array}{ll}\overline{\mathbf{H}} & \overline{\mathbf{H}}\end{array}\right]$,

$\mathbf{H}_{\mathbf{H H}}=\left[\begin{array}{ll}\mathbf{H}_{\mathbf{H}} & \mathbf{H}_{\mathbf{H}}\end{array}\right], \mathbf{P}_{\mathbf{P}}=\operatorname{diag}\{\mathbf{P}, \mathbf{P}\}$ and $\overline{\mathbf{M}}_{i j}, \overline{\mathbf{Y}}_{i j}, \mathbf{Y}_{i j}, \overline{\mathbf{M}}_{\mathbf{E} i j}$, $\mathbf{Y}_{\mathbf{E} i j}, \overline{\mathbf{Y}}_{\mathbf{E} i j}, \mathbf{T}_{i i}$ and $\mathbf{T}_{i j}$ are defined as (20). According to the (23), the (22) can be rewritten as follows:

$$
L V(x(t)) \leq \bar{x}^{\mathrm{T}}\left(\sum_{i=1}^{3} h_{i}^{2}\left[\begin{array}{cc}
\boldsymbol{\Lambda}_{11} & \boldsymbol{\Lambda}_{21}^{\mathrm{T}} \\
\boldsymbol{\Lambda}_{21} & \boldsymbol{\Lambda}_{22}
\end{array}\right]+2 \sum_{i=1, j<i}^{3} h_{i} h_{j}\left[\begin{array}{cc}
\tilde{\boldsymbol{\Lambda}}_{11} & \tilde{\boldsymbol{\Lambda}}_{21}^{\mathrm{T}} \\
\tilde{\boldsymbol{\Lambda}}_{21} & \tilde{\boldsymbol{\Lambda}}_{22}
\end{array}\right]\right) \bar{x}
$$

Integrating both side of (24) form 0 to $t, \forall t>0$ and then taking expectation with zero initial conditions, one has:

$$
\begin{aligned}
& E\{V(x(t))\} \leq E\left\{\int _ { 0 } ^ { t } \left(\sum_{i=1}^{3} h_{i}^{2} \bar{x}^{\mathrm{T}}\left[\begin{array}{cc}
\boldsymbol{\Lambda}_{11} & \boldsymbol{\Lambda}_{21}^{\mathrm{T}} \\
\boldsymbol{\Lambda}_{21} & \boldsymbol{\Lambda}_{22}
\end{array}\right] \bar{x}+2 \sum_{i=1, j<i}^{3} h_{i} h_{j}\right.\right. \\
& \left.\left.\times \bar{x}^{\mathrm{T}}\left[\begin{array}{cc}
\tilde{\boldsymbol{\Lambda}}_{11} & \tilde{\boldsymbol{\Lambda}}_{21}^{\mathrm{T}} \\
\tilde{\boldsymbol{\Lambda}}_{21} & \tilde{\boldsymbol{\Lambda}}_{22}
\end{array}\right] \bar{x}\right) d t\right\}
\end{aligned}
$$

Defining the following performance function for nonzero external disturbance i.e., $v(t) \neq 0$.

$$
\begin{aligned}
\Gamma(t) & =E\left\{\int_{0}^{t}\left(\gamma v^{\mathrm{T}}(t) v(t)-2 z^{\mathrm{T}}(t) v(t)\right) d t\right\} \\
& =E\left\{\int_{0}^{t}\left(\gamma v^{\mathrm{T}}(t) v(t)-2 z^{\mathrm{T}}(t) v(t)+L V(x(t))\right) d t-V(x(t))\right\} \\
& \leq E\left\{\int_{0}^{t}\left(\gamma v^{\mathrm{T}}(t) v(t)-2 z^{\mathrm{T}}(t) v(t)+L V(x(t))\right) d t\right\} \\
& \leq E\left\{\int_{0}^{t} \Psi(t) d t\right\}
\end{aligned}
$$

where

$$
\Psi(t)=\gamma v^{\mathrm{T}}(t) v(t)-2 z^{\mathrm{T}}(t) v(t)+L V(x(t))
$$

Substituting the (24) and (8b) into (27), one has:

$$
\begin{gathered}
\Psi(t) \leq \bar{x}^{\mathrm{T}}\left(\sum_{i=1}^{3} h_{i}^{2}\left[\begin{array}{cc}
\boldsymbol{\Lambda}_{11} & \boldsymbol{\Lambda}_{12}-\mathbf{C}_{i}^{\mathrm{T}} \\
* & \boldsymbol{\Lambda}_{22}+\gamma \mathbf{I}-\mathbf{D}_{i}^{\mathrm{T}}-\mathbf{D}_{i}
\end{array}\right]+2 \sum_{i=1, j<i}^{3} h_{i} h_{j}\right. \\
\left.\times\left[\begin{array}{cc}
\tilde{\boldsymbol{\Lambda}}_{11} & \tilde{\boldsymbol{\Lambda}}_{12}-\left(\mathbf{C}_{i}+\mathbf{C}_{j}\right)^{\mathrm{T}} / 2 \\
* & \tilde{\boldsymbol{\Lambda}}_{22}+\gamma \mathbf{I}-\left(\mathbf{D}_{i}^{\mathrm{T}}+\mathbf{D}_{i}+\mathbf{D}_{j}^{\mathrm{T}}+\mathbf{D}_{j}\right)^{\mathrm{T}} / 2
\end{array}\right]\right) \bar{x}
\end{gathered}
$$

If the condition (17b) is held then the (28) can be rewritten as follows:

$$
\begin{aligned}
& \Psi(t) \leq \bar{x}^{\mathrm{T}}\left(\sum_{i=1}^{3} h_{i}^{2}\left[\begin{array}{cc}
\boldsymbol{\Lambda}_{11} & \boldsymbol{\Lambda}_{21}^{\mathrm{T}}-\mathbf{C}_{i}^{\mathrm{T}} \\
\boldsymbol{\Lambda}_{21}-\mathbf{C}_{i} & \boldsymbol{\Lambda}_{22}+\gamma \mathbf{I}-\mathbf{D}_{i}^{\mathrm{T}}-\mathbf{D}_{i}
\end{array}\right]\right. \\
& \left.\quad+2 \sum_{i=1, j<i}^{3} h_{i} h_{j} \mathbf{Q}\right) \bar{x}
\end{aligned}
$$

From the relaxed concept of Lemma 2, one has:

$$
\Psi(t) \leq \sum_{i=1}^{3} h_{i}^{2} \bar{x}^{\mathrm{T}} \boldsymbol{\Lambda} \bar{x}
$$

Obviously, if the stability condition (17a) hold then the (30) is semi-negative i.e., $\Psi(t) \leq 0$. According to (26), we have follows:

$$
\Gamma(t) \leq 0
$$




$$
E\left\{2 \int_{0}^{t} z^{\mathrm{T}}(t) v(t) d t\right\} \geq E\left\{\gamma \int_{0}^{t} v^{\mathrm{T}}(t) v(t) d t\right\}
$$

Since (32) is equivalent to (14) of Definition 1, one can find that the uncertain T-S fuzzy model with multiplicative noise (11) is strictly input passive with external disturbance input and controlled output. Next, we will show the system is mean square stable which is necessary requirement performance. Based on the (30), if the condition (17a) of Theorem 1 is held, thus $\Psi(t) \leq 0$. By assuming the external disturbance $v(t)=0$, we can find $\Gamma(t) \leq 0$ from $(26)$ due to $\Psi(t) \leq 0$. And then, one has

$$
L V(x(t)) \leq 0
$$

Thus, the closed-loop uncertain T-S fuzzy model with multiplicative noise driven by the fuzzy controller (10) is mean square stable. The proof of this theorem is complement. \#

Noting that (17) not belongs to LMI problem, one cannot apply with convex optimization algorithm to solve it. For obtaining feasible solutions of Theorem 1, the LMI stability condition of next theorem was developed by some conversion techniques from (17).

\section{Theorem 2}

Given dissipative tare $\gamma>0$ and number of fired rule $s>1$, if there exits $\mathbf{P}=\mathbf{P}^{\mathrm{T}}>0, \mathbf{Q}=\mathbf{Q}^{\mathrm{T}}>0, \varepsilon_{i i}>0, \varepsilon_{i j}>0$ and feedback gain $\mathbf{F}_{i}$ to satisfy the following LMI relaxed stability conditions, then the uncertain T-S fuzzy model with multiplicative noise is mean square stable and strictly input passive.

$$
\mathbf{\Pi} \equiv\left[\begin{array}{ccccc}
\boldsymbol{\Pi}_{11} & \mathbf{E}_{i}-\mathbf{X C}_{i}^{\mathrm{T}} & \left(\mathbf{R}_{i} \mathbf{X}_{1}+\mathbf{R}_{B i} \mathbf{K}_{i}\right)^{\mathrm{T}} & 0 & \boldsymbol{\Pi}_{15} \\
* & \gamma \mathbf{I}-\mathbf{D}_{i}^{\mathrm{T}}-\mathbf{D}_{i} & 0 & \mathbf{R}_{\mathbf{E} i}^{\mathrm{T}} & \boldsymbol{\Pi}_{25} \\
* & * & -\varepsilon_{i i} \mathbf{I} & 0 & 0 \\
* & * & * & -\varepsilon_{i i} \mathbf{I} & 0 \\
* & * & * & * & \boldsymbol{\Pi}_{33}
\end{array}\right] \leq 0,
$$

$\tilde{\mathbf{\Pi}} \equiv\left[\begin{array}{ccccc}\tilde{\mathbf{\Pi}}_{11} & \mathbf{E}_{i j}-\mathbf{X}\left(\mathbf{C}_{i}^{\mathrm{T}}+\mathbf{C}_{j}^{\mathrm{T}}\right) / 2 & \tilde{\mathbf{\Pi}}_{13} & 0 & \tilde{\mathbf{\Pi}}_{15} \\ * & \gamma \mathbf{I}-\left(\mathbf{D}_{i}^{\mathrm{T}}+\mathbf{D}_{i}+\mathbf{D}_{j}^{\mathrm{T}}+\mathbf{D}_{j}\right) / 2 & 0 & \mathbf{Y}_{\mathbf{E} i j}^{\mathrm{T}} & \tilde{\mathbf{\Pi}}_{25} \\ * & * & \tilde{\mathbf{\Pi}}_{33} & 0 & 0 \\ * & * & * & \tilde{\mathbf{\Pi}}_{44} & 0 \\ * & * & * & * & \tilde{\mathbf{\Pi}}_{55}\end{array}\right] \leq 0$,

$$
\text { for } i \neq j \quad \forall j<i(34 \mathrm{~b})
$$

where

$$
\boldsymbol{\Pi}_{11}=\left(\mathbf{A}_{i} \mathbf{X}+\mathbf{B}_{i} \mathbf{K}_{i}\right)^{\mathbf{T}}+\left(\mathbf{A}_{i} \mathbf{X}+\mathbf{B}_{i} \mathbf{K}_{i}\right)+2 \varepsilon_{i i} \mathbf{H} \mathbf{H}^{\mathrm{T}}+(s-1) \hat{\mathbf{Q}}
$$

$$
\begin{gathered}
\boldsymbol{\Pi}_{15}=\left[\begin{array}{cc}
\left(\overline{\mathbf{A}}_{i} \mathbf{X}_{1}+\overline{\mathbf{B}}_{i} \mathbf{K}_{i}\right)^{\mathrm{T}} & \left(\overline{\mathbf{R}}_{i} \mathbf{X}_{1}+\overline{\mathbf{R}}_{B i} \mathbf{K}_{i}\right)^{\mathrm{T}}
\end{array}\right] \\
\boldsymbol{\Pi}_{25}=\left[\begin{array}{ll}
\overline{\mathbf{E}}_{i}^{\mathrm{T}} & \overline{\mathbf{R}}_{\mathbf{E} i}^{\mathrm{T}}
\end{array}\right] \\
\boldsymbol{\Pi}_{33}=\operatorname{diag}\left\{\boldsymbol{\varepsilon}_{i i} \overline{\mathbf{H}} \overline{\mathbf{H}}^{\mathrm{T}}-\mathbf{X},-\boldsymbol{\varepsilon}_{i i} \mathbf{I}\right\}
\end{gathered}
$$

$$
\begin{aligned}
\tilde{\boldsymbol{\Pi}}_{11}= & \left(\mathbf{A}_{i} \mathbf{X}+\mathbf{B}_{i} \mathbf{K}_{j}+\mathbf{A}_{j} \mathbf{X}+\mathbf{B}_{j} \mathbf{K}_{i}\right)^{\mathbf{T}} / 2+\left(\mathbf{A}_{i} \mathbf{X}+\mathbf{B}_{i} \mathbf{K}_{j}\right. \\
& \left.+\mathbf{A}_{j} \mathbf{X}+\mathbf{B}_{j} \mathbf{K}_{i}\right) / 2+4 \varepsilon_{i j} \mathbf{H} \mathbf{H}^{\mathrm{T}}-\hat{\mathbf{Q}}
\end{aligned}
$$

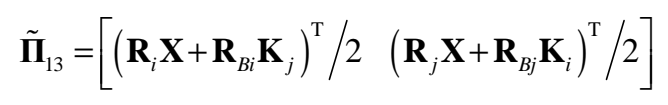

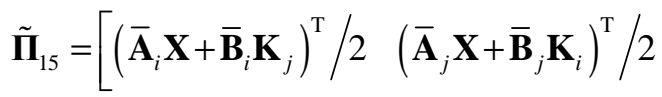

$$
\begin{aligned}
& \left.\left(\overline{\mathbf{R}}_{i} \mathbf{X}+\overline{\mathbf{R}}_{B i} \mathbf{K}_{j}\right)^{\mathrm{T}} / 2\left(\overline{\mathbf{R}}_{j} \mathbf{X}+\overline{\mathbf{R}}_{B j} \mathbf{K}_{i}\right)^{\mathrm{T}} / 2\right]
\end{aligned}
$$

$$
\tilde{\boldsymbol{\Pi}}_{25}=\left[\begin{array}{ll}
\overline{\mathbf{M}}_{\mathbf{E} i j}^{\mathrm{T}} & \overline{\mathbf{Y}}_{\mathbf{E} i}^{\mathrm{T}}
\end{array}\right]
$$

$$
\tilde{\mathbf{\Pi}}_{33}=\tilde{\mathbf{\Pi}}_{44}=\operatorname{diag}\left\{-\mathcal{E}_{i j} \mathbf{I},-\varepsilon_{i j} \mathbf{I}\right\}
$$

$$
\tilde{\mathbf{\Pi}}_{55}=\operatorname{diag}\left\{\left(\varepsilon_{i j} \overline{\mathbf{H}} \overline{\mathbf{H}}^{\mathrm{T}}-\mathbf{X}_{1}\right),\left(\varepsilon_{i j} \overline{\mathbf{H}} \overline{\mathbf{H}}^{\mathrm{T}}-\mathbf{X}_{1}\right),-\boldsymbol{\varepsilon}_{i j} \mathbf{I},-\varepsilon_{i j} \mathbf{I}\right\}
$$

and $\mathbf{X}=\mathbf{P}^{-1}, \hat{\mathbf{Q}}=\mathbf{P}^{-1} \mathbf{Q} \mathbf{P}^{-1}, \quad \mathbf{K}_{i}=\mathbf{F}_{i} \mathbf{P}^{-1}$

\section{Proof:}

First, multiplying the both side of $\boldsymbol{\Lambda}(17 \mathrm{a})$ by $\operatorname{diag}\left\{\mathbf{P}^{-1}, \mathbf{I}\right\}$, we have the following inequalities.

$$
\Xi=\left[\begin{array}{cc}
\boldsymbol{\Xi}_{11} & \boldsymbol{\Xi}_{12} \\
* & \boldsymbol{\Xi}_{22}
\end{array}\right] \leq 0
$$

where

$$
\begin{aligned}
\boldsymbol{\Xi}_{11}= & \left(\mathbf{A}_{i} \mathbf{P}^{-1}+\mathbf{B}_{i} \mathbf{F}_{i} \mathbf{P}^{-1}\right)^{\mathrm{T}}+\left(\mathbf{A}_{i} \mathbf{P}^{-1}+\mathbf{B}_{i} \mathbf{F}_{i} \mathbf{P}^{-1}\right)+2 \mathbf{H} \mathbf{H}^{\mathrm{T}}+\varepsilon_{i i}^{-1} \\
& \times\left(\mathbf{R}_{i} \mathbf{P}^{-1}+\mathbf{R}_{\mathbf{B} i} \mathbf{F}_{i} \mathbf{P}^{-1}\right)^{\mathrm{T}}\left(\mathbf{R}_{i} \mathbf{P}^{-1}+\mathbf{R}_{\mathbf{B} i} \mathbf{F}_{i} \mathbf{P}^{-1}\right) \\
& +\left(\overline{\mathbf{A}}_{i} \mathbf{P}^{-1}+\overline{\mathbf{B}}_{i} \mathbf{F}_{i} \mathbf{P}^{-1}\right)^{\mathrm{T}} \mathbf{T}_{i i}\left(\overline{\mathbf{A}}_{i} \mathbf{P}^{-1}+\overline{\mathbf{B}}_{i} \mathbf{F}_{i} \mathbf{P}^{-1}\right) \\
& +\varepsilon_{i i}^{-1}\left(\overline{\mathbf{R}}_{i} \mathbf{P}^{-1}+\overline{\mathbf{R}}_{\mathbf{B} i} \mathbf{F}_{i} \mathbf{P}^{-1}\right)^{\mathrm{T}}\left(\overline{\mathbf{R}}_{i} \mathbf{P}^{-1}+\overline{\mathbf{R}}_{\mathbf{B} i} \mathbf{F}_{i} \mathbf{P}^{-1}\right) \\
& +(s-1) \mathbf{P}^{-1} \mathbf{Q} \mathbf{P}^{-1}
\end{aligned}
$$




$$
\begin{aligned}
\mathbf{\Xi}_{12}= & \mathbf{E}_{i}-\mathbf{P}^{-1} \mathbf{C}_{i}^{\mathrm{T}}+\left(\overline{\mathbf{A}}_{i} \mathbf{P}^{-1}+\overline{\mathbf{B}}_{i} \mathbf{F}_{i} \mathbf{P}^{-1}\right)^{\mathrm{T}} \mathbf{T}_{i i} \overline{\mathbf{E}}_{i} \\
& +\varepsilon_{i i}^{-1}\left(\overline{\mathbf{R}}_{i} \mathbf{P}^{-1}+\overline{\mathbf{R}}_{\mathbf{B} i} \mathbf{F}_{i} \mathbf{P}^{-1}\right)^{\mathrm{T}} \overline{\mathbf{R}}_{\mathbf{E} i}
\end{aligned}
$$

$$
\boldsymbol{\Xi}_{22}=\varepsilon_{i i}^{-1} \mathbf{R}_{\mathbf{E} i}^{\mathrm{T}} \mathbf{R}_{\mathbf{E} i}+\overline{\mathbf{E}}_{i}^{\mathrm{T}} \mathbf{T}_{i i} \overline{\mathbf{E}}_{i}+\varepsilon_{i i}^{-1} \overline{\mathbf{R}}_{\mathbf{E} i}^{\mathrm{T}} \overline{\mathbf{R}}_{\mathbf{E} i}+\gamma \mathbf{I}-\mathbf{D}_{i}^{\mathrm{T}}-\mathbf{D}_{i}
$$

By setting the $\mathbf{X}=\mathbf{P}^{-1}, \hat{\mathbf{Q}}=\mathbf{P}^{-1} \mathbf{Q} \mathbf{P}^{-1}$ and $\mathbf{K}_{i}=\mathbf{F}_{i} \mathbf{P}^{-1}$ and applying the Schur complement in Tanaka and Wang [15], the inequality (38) can be converted into the same as LMI relaxed stability condition (34a) i.e. $\Pi \leq 0$ for $i=j$. Besides, the proof of condition (34b) $\tilde{\boldsymbol{\Pi}} \leq 0$ for $i \neq j$ can be found with the same process of condition (34a). Hence, we have omitted the proof of condition (34b) here and the proof was complement.\#

Obviously, if the LMI-based relaxed stability conditions (34) can be satisfied by suitable solutions as $\mathbf{X}, \hat{\mathbf{Q}}$ and $\mathbf{M}_{i}$, then we can find the feasible solutions for satisfying relaxed stability conditions (17) by $\mathbf{P}=\mathbf{X}^{-1}, \mathbf{Q}=\mathbf{P} \hat{\mathbf{Q}} \mathbf{P}$ and $\mathbf{F}_{i}=\mathbf{K}_{i} \mathbf{P}$. Based on the feasible solutions, one can find that the uncertain T-S fuzzy model with multiplicative noise can be achieved mean square stable and strictly input passive by designed fuzzy controller. Accordingly, the proposed design technique can be applied to design the fuzzy controller for stabilizing the nonlinear drum-boiler system under perturbed and disturbed. In the next section, the simulation results were presented to manifest the effectively and applications of the proposed controller design technique.

\section{FUZZY CONTROLLER DESIGN AND SIMULATION RESULTS}

With the T-S fuzzy model (7) of nonlinear drum-boiler system (5), the proposed fuzzy controller design technique of this paper was applied in this section. The uncertainties and stochastic behaviors of nonlinear drum-boiler system (5) are assumed as follows:

\section{Uncertainty Parameters:}

$\overline{\mathbf{A}}_{i}=\overline{\mathbf{A}}=\operatorname{diag}\{0,0.1,0,0\}, \overline{\mathbf{B}}_{i}=0, \overline{\mathbf{E}}_{i}=\overline{\mathbf{E}}=0.1 \mathbf{E}_{i}$,

$\mathbf{H}=0.1 \mathbf{I}, \quad \overline{\mathbf{H}}=0.001 \mathbf{H}$ and $\Delta_{i}(t)=\bar{\Delta}_{i}(t)=\sin (5 t)$

\section{Multiplicative Noise Parameters:}

$\mathbf{R}_{i}$ are chosen as the first and second rows be $1 \%$ multiples the corresponding elements of $\mathbf{A}_{i}, \mathbf{R}_{\mathbf{B} i}=0, \mathbf{R}_{\mathbf{E} i}=0.1 \mathbf{E}_{i}, \overline{\mathbf{R}}_{i}=$ $\overline{\mathbf{R}}=0.1 \sum_{i=1}^{3} \mathbf{R}_{i} / 3, \quad \overline{\mathbf{R}}_{\mathbf{B} i}=0$ and $\overline{\mathbf{R}}_{\mathbf{E} i}=\overline{\mathbf{R}}_{\mathbf{E}}=0.1 \sum_{i=1}^{3} \mathbf{R}_{\mathbf{E} i} / 3$.

Besides, the matrices of output $z(t)$ are chosen as $\mathbf{C}_{i}=$ $\left[\begin{array}{llll}0 & 1 & 0 & 0\end{array}\right]$ and $\mathbf{D}_{i}=1$ to achieve the passivity definition of
Definition 1. In addition, the number of fired rule is chosen as $s=2$ due to the membership function, and the dissipative rate $\gamma=1$ is chosen for determining the level of attenuation performance. According to the above matrices, the final uncertain T-S fuzzy model is thus described as follows:

\section{Plant Part:}

$$
\begin{gathered}
\dot{x}(t)=\sum_{i=1}^{3} h_{i}\left(x_{1}(t)\right)\left\{\left(f_{i}(t)+\Delta f_{i}(t)\right)+(g(t)+\Delta g(t)) \beta(t)\right\} \\
z(t)=\sum_{i=1}^{3} h_{i}\left(x_{1}(t)\right)\left\{\mathbf{C}_{i} x(t)+\mathbf{D}_{i} v(t)\right\}
\end{gathered}
$$

where

$$
\begin{gathered}
f_{i}(t)=\mathbf{A}_{i} x(t)+\mathbf{B}_{i} u(t)+\mathbf{E}_{i} v(t) \text { and } \\
\Delta f_{i}(t)=\mathbf{H} \Delta(t)\left(\mathbf{R}_{i} x(t)+\mathbf{R}_{\mathbf{E} i} v(t)\right),
\end{gathered}
$$

$g(t)=\overline{\mathbf{A}} x(t)+\overline{\mathbf{E}} v(t)$ and $\Delta g(t)=\overline{\mathbf{H}} \bar{\Delta}(t)\left(\overline{\mathbf{R}} x(t)+\overline{\mathbf{R}}_{\mathbf{E}} v(t)\right)$,

for $i=1,2,3$. Based on the fuzzy model (41), the proposed design technique is applied to find the feasible solutions via LMI-based stability conditions (34). By using the LMI Toolbox of MATLAB, the feasible solutions of (34) can be obtained as follows:

$\mathbf{P}=\left[\begin{array}{cccc}0.0125 & 0 & 0 & 0.0049 \\ 0 & 0.1153 & 0 & 0 \\ 0 & 0 & 0.0133 & 0 \\ 0.0049 & 0 & 0 & 0.0063\end{array}\right]$,

$\mathbf{Q}=\left[\begin{array}{cccc}0.0097 & -0.0001 & -0.0002 & 0.0042 \\ -0.0001 & 0.9912 & 0 & 0 \\ -0.0002 & 0 & 0.0131 & -0.0001 \\ 0.0042 & 0 & -0.0001 & 0.0018\end{array}\right]$

$\mathbf{F}_{1}=\left[\begin{array}{cccc}-3.0007 & 0.337 & -0.0409 & -1.3142 \\ -2.9584 & -1.2537 & -0.0403 & -1.2957 \\ 0.0456 & 0.0008 & -1.3643 & 0.0041\end{array}\right]$,

$\mathbf{F}_{2}=\left[\begin{array}{cccc}-2.9663 & 0.2659 & -0.1747 & -0.6918 \\ -2.9242 & -1.3238 & -0.1713 & -0.6821 \\ 0.0989 & -0.0802 & -1.3642 & 0.8009\end{array}\right]$ and 


$$
\begin{gathered}
\mathbf{F}_{3}=\left[\begin{array}{cccc}
-2.8935 & 0.182 & -0.3103 & 0.0625 \\
-2.8521 & -1.3982 & -0.3041 & 0.0615 \\
0.1538 & -0.1689 & -1.3537 & 1.5995
\end{array}\right], \\
\varepsilon_{11}=74.7542, \varepsilon_{22}=74.2927 \text {, and } \varepsilon_{33}=16.2104 .
\end{gathered}
$$

According to Theorem 2, the solutions of (43) can also satisfy the stability conditions (17) that mean the systems can achieve mean square stable and strictly input passive via fuzzy controller (44). Via the concept of PDC in Tanaka and Wang [15], the following PDC-based fuzzy controller can be designed with feedback gains $\mathbf{F}_{i}$ for $i=1,2,3$ defined in (43).

\section{Controller Part:}

$$
u(t)=\sum_{i=1}^{3} h_{i}\left(\mathbf{F}_{i} x(t)\right)
$$

The responses of system can be simulated by SimuLink Toolbox of MATLAB for verifying the effectiveness and application of proposed design approach. By using the designed fuzzy controller (44), the uncertain stochastic T-S fuzzy system (8) with multiplicative noise can be stabilized as the offered Figs. 3-6 with initial condition $x(0)=\left[\begin{array}{llll}20 & 2.75 & 550 & 1\end{array}\right]^{\mathrm{T}}$.

Besides, the external disturbance $v(t)$ is chosen as a zeromean white noise with variance 0.1 . In Figs. 3-4, one can find that the drum pressure $y_{1}(t)$ and excess oxygen level $y_{2}(t)$ are kept on 320 PSI and near 2.5 percent, respectively, to maintain the operating conditions of boiler. The Figs. 3-4 also show the good accuracy of drum pressure and excess oxygen level both in short term and long term characteristics. In Fig. 4, there appears some vibrations in simulation process that caused by the external disturbance $v(t)$. According to the fuzzy controller (44), the effect of external disturbance on system is constrained by passivity property (14) for maintaining the system stability. Besides, the Figs. 5 and 6 show a long time response for convergence. The drum water level and steam flow rate are also controlled on the argument points in simulation time. Thus, according to the simulation results, one can find that the nonlinear drum-boiler system (1) with uncertainties and multiplicative noise (43) can be stabilized in argument points via the designed fuzzy controller (44). Moreover, in order to check the achievement of strict input passivity of (14), the value of following ratio can be obtained from simulation results.

$$
\frac{E\left\{2 \int_{0}^{t} z^{\mathrm{T}}(t) v(t) d t\right\}}{E\left\{\int_{0}^{t} v^{\mathrm{T}}(t) v(t) d t\right\}}=1.998
$$

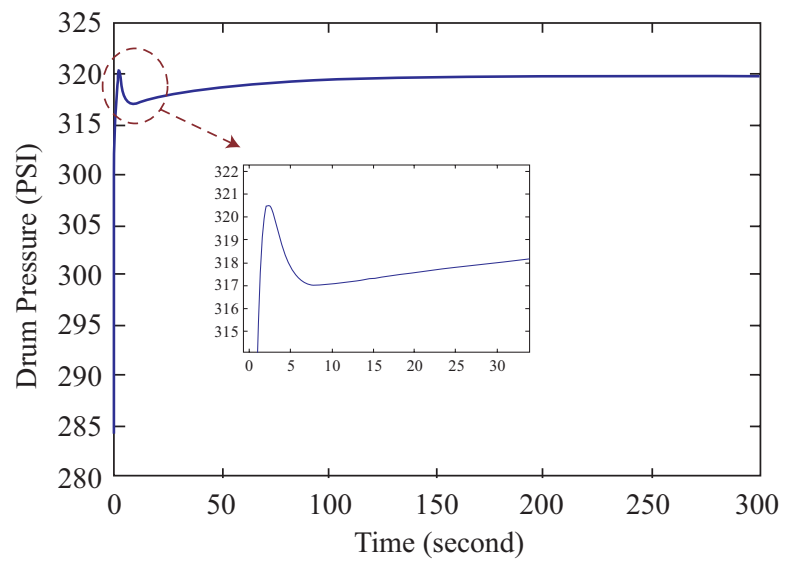

Fig. 3. Responses of Measured Drum Pressure $y_{1}(t)$.

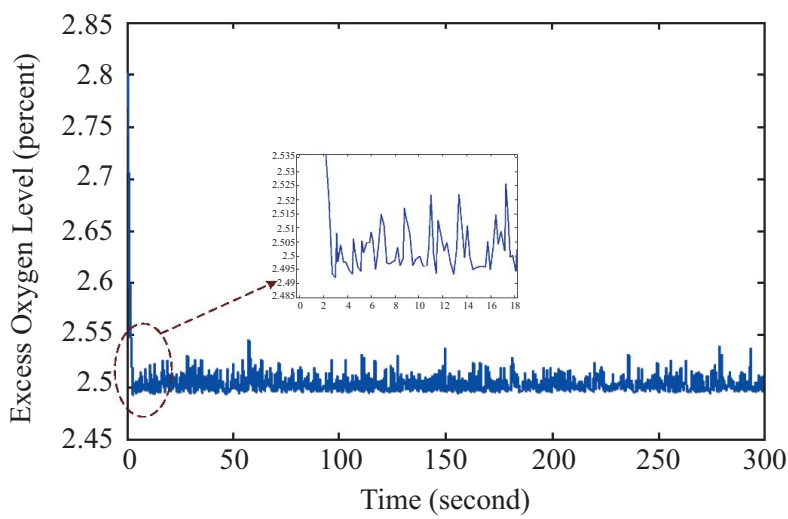

Fig. 4. Responses of Excess Oxygen Level $y_{2}(t)$.

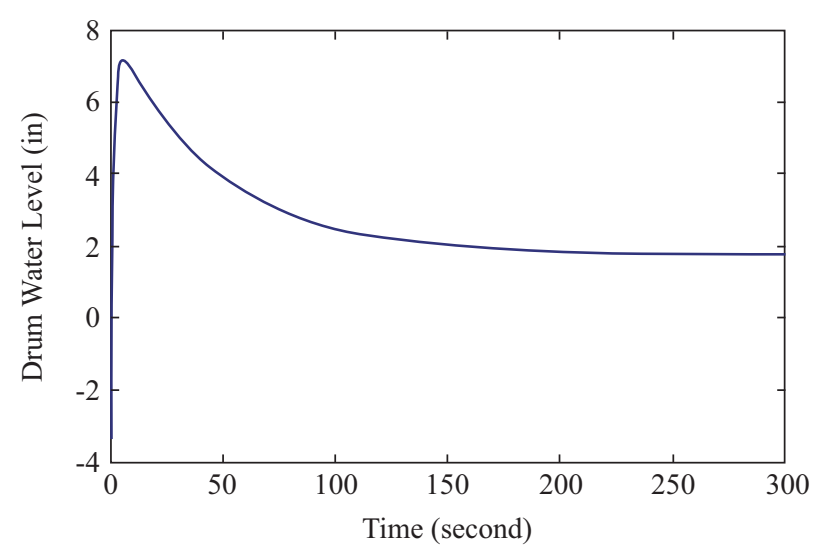

Fig. 5. Responses of Drum Water Level $y_{3}(t)$.

Obviously, the ratio value of (45) is bigger than dissipative rate $\gamma=1$ that satisfies the Definition 1 . The considered system is thus strictly input passive driven by the designed fuzzy controller (44). Therefore, the considered system (41) controlled by the fuzzy controller (44) is mean square stable and strictly input passive. 


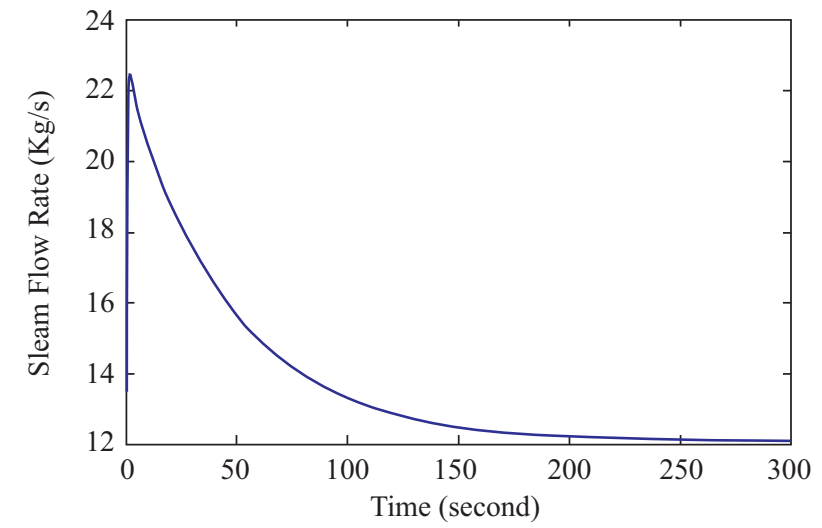

Fig. 6. Responses of Steam Flow Rate $y_{4}(t)$.

\section{CONCLUSIONS}

In this paper, we investigated the stability and stabilization problems of perturbed nonlinear drum-boiler system with multiplicative noises via T-S fuzzy model approach. We also considered the uncertainties and stochastic behaviors for simulating the varying of operating environment. The passivity theory was employed to discuss the effect of external disturbance on considered system for investigating the attenuation performance. Besides, the relaxed concept was employed to extend the region of feasible solutions for designing the fuzzy controller. According to the convex optimization algorithm, the relaxed stability and stabilization conditions can be converted into LMI forms. From the simulation results, one can find that the measured outputs were maintained with valid level for keeping the smooth working of boiler system. By employing the derivations of propose approach, one can extend the results of this paper into more complex systems in further discussions, for example timedelay or observer-based control systems.

\section{REFERENCES}

1. Abdeldjebar, B. and Khier, B., "Modeling, identification for the grade boiler a comparison fuzzy logic, PID and application of overheat steam temperature," Proceeding of International Conference on Control, Automation and Systems, pp. 256-261, Seoul, Korea (2007).

2. Åström, K. J. and Zcklund, K., "A simplified nonlinear model of a drum boiler-turbine unit," International Journal of Control, Vol. 16, No. 1, pp. 145-169 (1972).
3. Chang, W. J., Chang, W., and Liu, H. H., "Model-based fuzzy modeling and control for autonomous underwater vehicles in the horizontal plane," Journal of Marine Science and Technology, Vol. 11, No. 3, pp. 155-163 (2003).

4. Chang, W. J., Huang, H. D., and Liang, H. J., "T-S fuzzy controller design for a benchmark drum-boiler system," Proceeding of 13th National Conference on Fuzzy Theory and Its Applications, p. 44, Kaohsiung, Taiwan (2005).

5. Chang, W. J., Ku, C. C., Chang, W., and Huang, P. H., "Passive fuzzy control with relaxed conditions for discrete affine T-S fuzzy systems," International Journal of Innovative Computing, Information and Control, Vol. 3, No. 4, pp. 853-871 (2007).

6. Chang, W. J. and Shing, C. C., "Robust covariance control for discrete system by Takagi-Sugeno fuzzy controllers," ISA Transactions, Vol. 43, No. 3, pp. 377-387 (2004).

7. Chawdry, P. K. and Hogg, B. W., "Identification of boiler models," IEE Proceedings D Control Theory \& Applications, Vol. 136, No. 5, pp. 261-271 (1989).

8. Eli, G., Uri, S., and Isaac, Y., $H_{\infty}$ Control and Estimation of State-Multiplicative Linear Systems, Springer, London (2005).

9. Fang, F., Liu, J., and Tan, W., "Output tracking control of a nonlinear boiler-turbine unit," Proceeding of IEEE Conference Decision and Control, Vol. 3, pp. 2615-2620, Paradise Island, Bahamas (2004).

10. Ho, D. W. C. and Niu, Y., "Robust fuzzy design for nonlinear uncertain stochastic systems via sliding-model control," IEEE Transactions on Fuzzy Systems., Vol. 15, No. 3, pp. 350-358 (2007).

11. Li, C., Zhang, H., and Liao, X., "Passivity and passification of fuzzy systems with time delays," Computers and Mathematics with Applications., Vol. 52, No. 6-7, pp. 1067-1078 (2006).

12. Lozano, R., Brogliato, B., Egeland, O., and Maschke, B., Dissipative Systems Analysis and Control Theory and Applications, Springer, London (2000).

13. Pellegrinetti, G. and Bentsman, J., "Nonlinear control oriented boiler modeling-a benchmark problem for controller design," IEEE Transactions on Control Systems Technology, Vol. 4, No. 1, pp. 57-64 (1996).

14. Tan, W., Marquez, H. J., and Chen, T., "Multivariable robust controller design for a boiler system," IEEE Transactions on Control Systems Technology, Vol. 10, No. 5, pp. 735-742 (2002).

15. Tanaka, K. and Wang, H. O., Fuzzy Control Systems Design and Analysis: A Linear Matrix Inequality Approach, John Wiley \& Sons, Inc., New York (2001).

16. Teixeira, M. C. M. and Zak, S. H., "Stabilizing controller design for uncertain nonlinear systems using fuzzy models," IEEE Transactions on Fuzzy Systems, Vol. 7, No. 2, pp. 133-142 (1999).

17. Wang, Z., Ho, D. W. C., and Liu, X., "A note on the robust stability of uncertain stochastic fuzzy systems with time-delays," IEEE Transactions on Systems, Man and Cybernetics-Part A: Systems and Humans, Vol. 34, No. 4, pp. 570-576 (2004).

18. Yang, H. J., "On the dissipativity of nonlinear systems: fuzzy control approach," Fuzzy Sets and Systems, Vol. 156, No. 2, pp. 185-207 (2005).

19. Zhang, H., Wang, Y., and Liu, D., "Delay-dependent guaranteed cost control for uncertain stochastic fuzzy systems with multiple time delays," IEEE Transactions on Systems, Man and Cybernetics-Part B: Cybernetics, Vol. 38, No. 1, pp. 126-140 (2008). 